\title{
An Integrated Surgical Suite Management Information System
}

\author{
James B. Martin, Ph.D., Mary E. Cantrell, M.D., \\ Robert G. Fichman, Phillip K. Hunsberger, and \\ Myra Radoyevich
}

\begin{abstract}
The operational aspects, application areas, and results achieved from an integrated surgical suite management information system are described. The system, which has been operating within Henry Ford Hospital in Detroit, Michigan, for 4 years, captures comprehensive data for each surgical episode, performs extensive edits on these data to assure data base integrity, and utilizes this data base in multiple applications. These applications include fixed-format reporting for medical staff and management; ad hoc retrieval capabilities to support research, education. and decision making; and linkage to other hospital systems to reduce both data redundancy and paper flow.
\end{abstract}

\section{INTRODUCTION}

Prompt access to accurate computerized data is essential to maintain and improve the overall performance of a surgical suite. For example, the surgically related functions of utilization monitoring, surgical case scheduling, patient billing, and retrospective medical studies all require readily accessible and correct data that can be manipulated easily. Large volumes of pertinent data are generated manually within a surgical suite in the form of patients' surgical records. A well-designed information system will capture a subset of these data in an efficient manner and generate, upon demand, information needed to support both medical and management functions. ORMIS (Operating Room Management Information System), designed for Henry Ford Hospital (HFH) in Detroit, Michigan, is such an information system. The purposes of this article are to describe the design, operation, and uses of ORMIS and to discuss lessons that have been learned during the implementation and operational phases of this system's life cycle.

From the University of Michigan and the Henry Ford Hospital. 


\section{PREVIOUSLY REPORTED WORK}

Given that the surgical suite is both a high-cost and high-revenue-generation area, one would expect considerable activity in surgically related MIS. Surprisingly few implemented systems, however, have been described in the literature. The majority of reported systems are batch-oriented and appear to have been developed primarily to provide for the automated generation of fixed format utilization reports on a periodic basis. ${ }^{1-9}$ These systems are, for the most part, not integrated with other hospital systems, although most are run on large main frame systems. Recently, in accordance with general technological developments in the field, systems with on-line data entry have been reported, ${ }^{10-12}$ and several of these have been integrated with other hospital systems (ADT, medical records abstract, and infection control). ${ }^{11-12}$

\section{ENVIRONMENT}

HFH is a large teaching hospital with 22 ORs in its surgical suite and a closed group-practice medical staff. The hospital performs all surgically related billing, including both surgery and anesthesiology professional fees. Surgically related staff include approximately 120 surgeons (distributed among 18 services), 23 anesthesiologists, 34 certified nurse anesthetists (CRNAs), 136 surgical residents, and 190 OR nursing personnel. ORMIS design and development work has been performed by the Management Information Systems Group (MISG) of the Program and Bureau of Hospital Administration, the University of Michigan, through an ongoing research agreement with HFH. The ongoing operation of ORMIS is the responsibility of the OR billing section, a group of four clericals reporting to the assistant administrator for the surgical suite.

\section{DATA CAPTURE}

The OR record (Figure 1) is used to record data for each surgical episode, serving as a legal, historical, and financial document. The current $O R$ record replaced two separate documents - one medically related and one financially related. Data collection is conducted in the OR during surgery, primarily by the circulating nurse, although as detailed below, some data are recorded by other OR staff members. The anesthetic record (Figure 2) is used to document the administration of anesthesia. This form is a legal document completed whenever a member of the professional anesthesia staff, either an anesthesiologist or a CRNA, is involved with a case. Like the OR record, the anesthetic record was designed to include both medical and financial data. Completed by the anesthesiologist and/or CRNA(s), the-anesthetic record follows the patient into the recovery room.

\section{COMPUTER SYSTEM OVERVIEW}

ORMIS is currently run on the Michigan Terminal System (MTS), the operating 


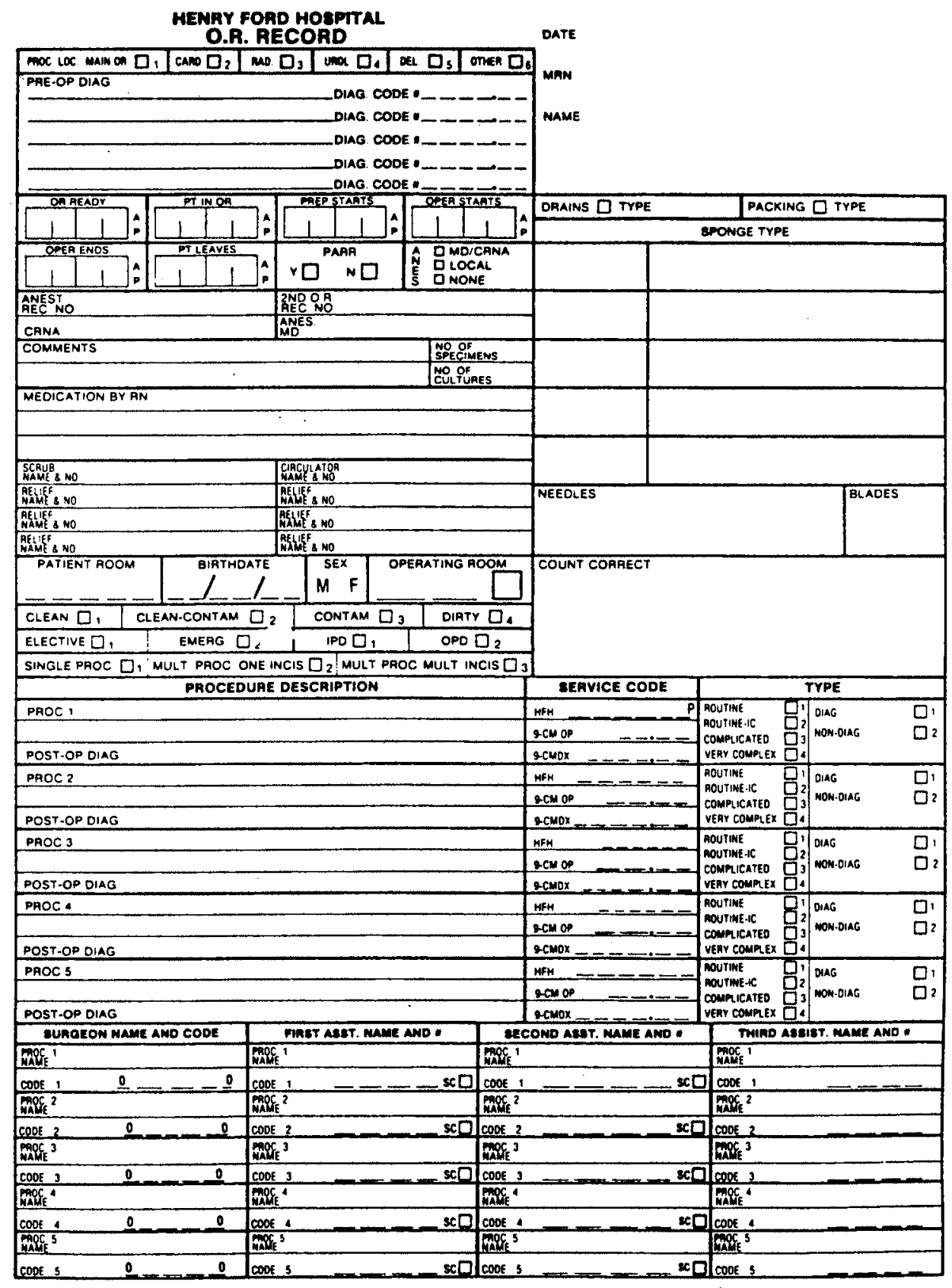

1. PATIENT ChaRT

Figure 1. Henry Ford Hospital operating room record. 
system for the University of Michigan's 16-megabyte Amdahl 5860 time-sharing computer. Data are entered on-line using a 1,200-baud CRT console chosen for its built-in software features, which support the construction and use of electronic "forms." ORMIS is composed of several large software modules that drive its major functions-data entry and editing, retrospective error detection and control, automatic patient charging, fixed format reports, and ad hoc data retrieval. The total software package includes customdesigned application programs, which total approximately 10,000 lines of PL/1 source code; a general subroutine library developed for use by ORMIS and other HFH systems; and an Information Storage and Retrieval System (ISRS) that also covers other HFH systems.

ORMIS accesses a number of supporting data files during both the input and output processes. Since data integrity is heavily dependent upon the ability to keep these files current, most are directly updatable in an on-line manner by OR personnel. The major support files include (1) HFH internal service code files and fee schedules; (2) ICD-9CM diagnostic and operative code files; (3) surgeon code files (including a unique code, multiple codes associated with different HFH facilities, division association, start and stop dates); (4) resident code files (including a unique code, legal specialties to which rotation is allowed, start and stop dates); and (5) nursing code files.

\section{CODING}

A number of coding activities are performed prior to data entry. Patients are coded using their MRNs; surgeons, anesthesiologists, nurses, CRNAs, and residents using HFH-assigned personnel codes; operative procedures using both an HFH internal coding scheme and ICD-9-CM operative codes; and diagnoses (both pre- and postoperative) using ICD-9-CM diagnostic codes. The HFH internal procedure codes, derived from the Medicare coding system, facilitate reporting and billing, while the ICD-9-CM procedure codes are used primarily by the medical records department. Electronic transfer of ICD9-CM procedure and diagnostic codes to the medical records system has replaced a once duplicate coding and data entry effort, which was performed within the medical records department. Surgeons, nurses, CRNAs, and residents are responsible for assuring that their codes are properly recorded on data collection documents during or immediately after surgery. ICD-9-CM diagnoses and procedures are coded by an accredited record technician (ART), who reports operationally to the medical records department but who has been assigned permanently to the surgical suite.

A unique system feature is that surgeons participate directly in the coding of operative procedures for billing purposes. An OR biller is stationed permanently at the main desk of the OR during normal working hours. Following case completion, the primary surgeon stops at the front desk, and HFH internal procedure codes are determined through a combined effort between the surgeon and the biller. The surgeon explains which procedures were performed while the biller looks up the most likely codes in a fee schedule; then together they decide which code(s) and fee(s) are most appropriate. This direct surgeon involvement is believed to be one of the key factors leading to the overall success of ORMIS. 


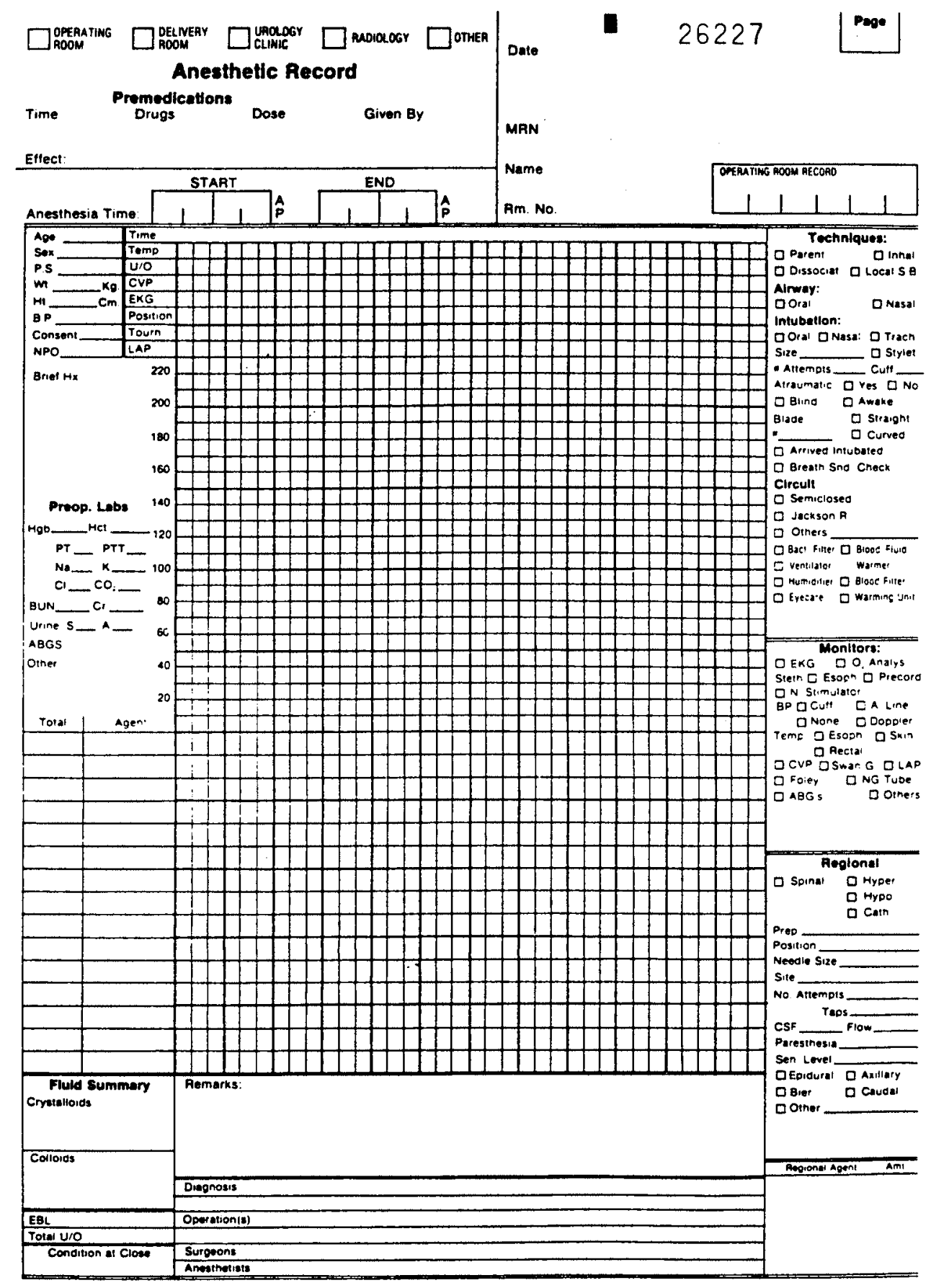

Figure 2. Henry Ford Hospital anesthetic record. 


\section{DATA ENTRY}

The ORMIS data entry function is decentralized - that is, records are entered in the surgical suite area rather than in a centrally located data processing department. Approximately 435 OR records are entered into the data base each week -270 from the OR and 165 total from five other areas that use ORMIS: the urology, general surgery, radiology (for vascular and neurosurgery procedures), and cardiology clinics, and the labor and delivery area (where all deliveries, both $\mathrm{C}$-section and vaginal, are recorded on the OR record). In addition, approximately 300 anesthesia records are entered each week.

ORMIS uses a forms-based data-entry procedure - the system creates on the terminal a facsimile of the document to be entered, leaving blank spaces for the data values to be keyed. Keyed records are sent to the data base after passing a series of sophisticated concurrent edits, which make accuracy and consistency checks on field values. Records that fail one or more edits remain on the screen with erroneous fields "flashing." Many times, these records can be corrected immediately and resubmitted, but if this is not possible they are "skipped" (i.e., sent to the data base with all detected errors noted within a set of "error bits" attached to each record). Error reports and other application programs are able to read a record's error bits, map them to associated error conditions, and take actions based on those conditions without having to repeat the original editing procedure. The error bits are used to minimize the occurrences of reporting data known to be in error.

\section{DATA INTEGRITY}

One of the important lessons learned during the implementation and operational phases of the ORMIS life cycle involves the heavy emphasis that must be placed on data integrity in systems that handle information on physician activities and research interests. While the system started with relatively simple edit routines, the initial feedback of data to the medical staff quickly indicated the need for more sophisticated edits as well as for improved control procedures to assure that all episodes are recorded and that all detected data errors are eventually corrected. Increasing use of ORMIS data for multiple purposes (as reported in the next section) has led over time to sophisticated data integrity procedures. The structure of these procedures is reported here since it should be useful to those building similar data-collection systems.

Data integrity maintenance has three component activities - error detection, correction, and control. Errors are detected concurrently during data entry as mentioned above, but retrospective error routines are also used to make consistency checks based upon information not available when records are originally entered. Retrospective error correction is performed on the same screens used for data entry by "fetching" targeted records to the screen, making required/changes, and resubmitting the records. The error control function involves identification of missing records and documentation of records containing known errors.

Almost 400 edit checks are made for each entered OR record, while the smaller anesthesia record undergoes approximately 70 checks. Concurrent "intrarecord" edits 
evaluate each record on its internal content. For example, surgery times are checked individually for illegal values, and as a group for nonsequential values. Additional concurrent checks include "intersystem" edits between the OR and census systems to verify that surgical patients designated as inpatients are active in the census system on the surgery date and to assure that those designated as outpatient are not active in the census system on the surgery date.

Two types of retrospective error checks complement concurrent editing: (1) time overlap routines make "intrasubsystem" checks between $O R$ record occurrences, and (2) cross-edit routines make "intersubsystem" checks between OR and anesthesia record occurrences. The time overlap routine detects most occurrences of improperly recorded surgery dates or specific operating rooms. The OR and anesthesia records belonging to a particular surgical episode share purposely designed redundant information (e.g., surgery date and MRN). Other interdependencies exist as well: (1) the OR record should "point" to the same anesthesia record that "points" back to it (through the anesthesia record number recorded on the OR record and vice versa); and (2) surgery times should fit chronologically with anesthesia times. Checks for these types of consistencies are performed by the cross-edit routines after all records of each type have been data-entered.

Medical, legal, and financial considerations require that all $O R$ cases be fully accounted for and documented. ORMIS assists this control process through use of the sequential document control number appearing on both the $O R$ record and the anesthesia record. The missing record routine identifies missing records, which are carefully monitored through manual coordination with the OR schedule, and voided documents (e.g., those damaged during the embossing process) are designated as such in the data base.

\section{APPLICATION AREAS}

\section{Fixed-Format Reports}

A central goal of ORMIS development has been to furnish comprehensive, yet concise and readable, fixed-format reports for use by both medical and administrative personnel. While a number of reports have been developed, only three of the most important are described here - the OR committee report package, the surgeon/resident/ department reports, and the undictated/unsigned report. These three report packages are all produced on a laser printer, allowing high-resolution output on letter-sized sheets.

\section{OR Committee Report Package}

This monthly package consists of three parts, which describe OR usage in different ways. The first section (Figure 3) contains OR percentage utilization statistics for each surgical service. HFH schedules surgical services in a blocked fashion within the 8:00 a.m. $-4: 00$ p.m., Monday-Friday, time period. Surgery times, which begin during this period but which end after 4:00 p.m., are referred to as "overruns." The three utilization percentages shown in Figure 3 are computed as (1) all elective case hours, with overruns; (2) all elective and emergency case hours, with overruns; (3) all elective and emergency case hours, excluding overruns. For each of these treatments, an approximated 20 minutes 


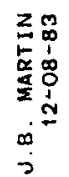

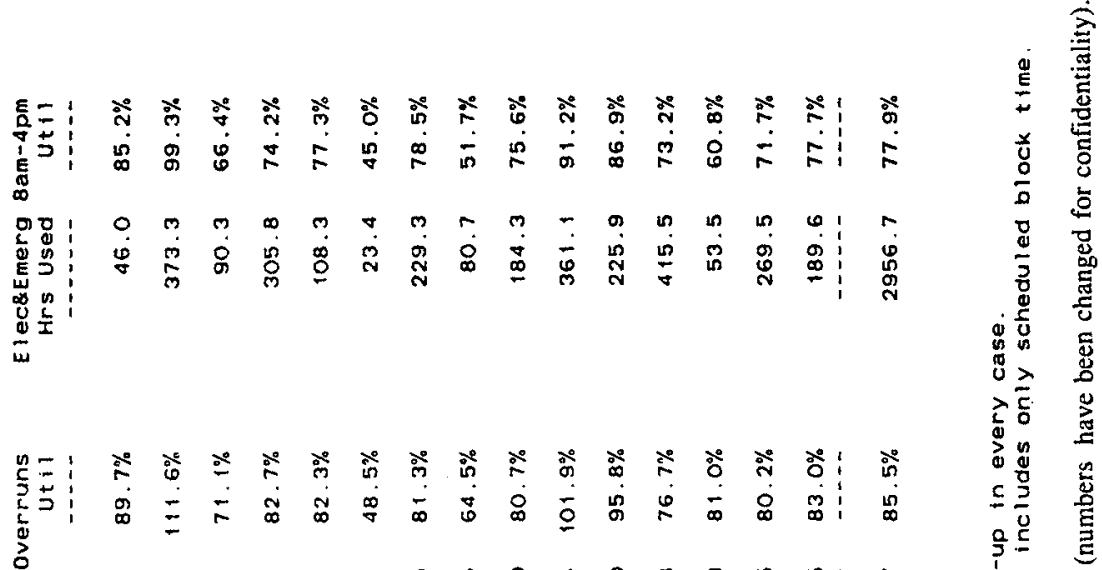

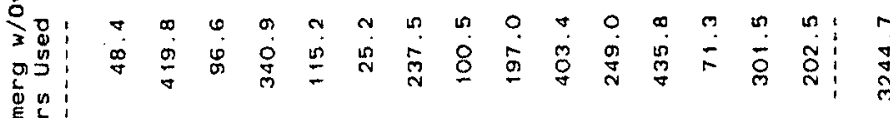

$$
\begin{aligned}
& \text { 岁主 }
\end{aligned}
$$

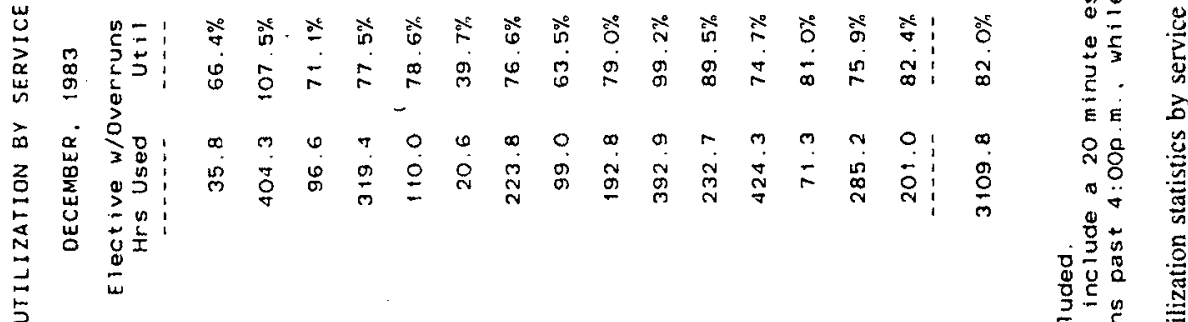

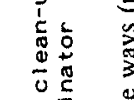

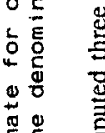

$$
\begin{aligned}
& \text { 芼告 } \\
& \text { 这 } \\
& \text { 要䔽 } \\
& \text { 论 }
\end{aligned}
$$

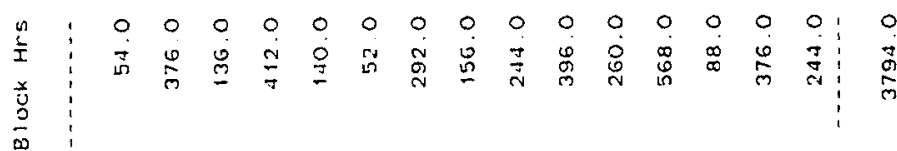

$$
\begin{aligned}
& \text { Xำ } \\
& \text { 0. जิ 造 }
\end{aligned}
$$

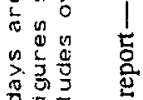

$$
\begin{aligned}
& \text { 政定 } \\
& \text { 옹 } \\
& \text { 西 } \\
& \text { 号军弯 }
\end{aligned}
$$

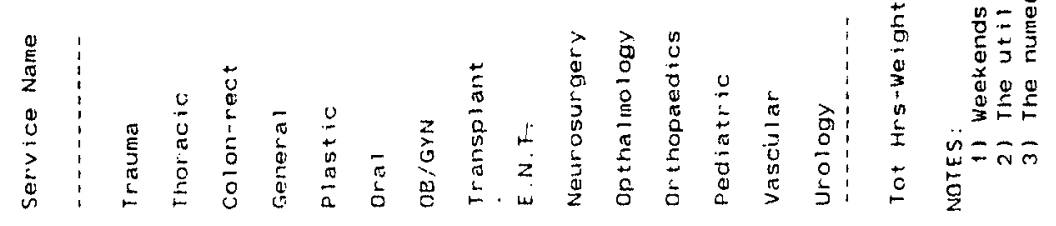


per case for cleanup time is added, and the denominator of the utilization figure is assigned block hours. Figure 3 provides comprehensive utilization data for the most recent month, while a second page provides a 12-month history of utilization for each service. The second section of the OR committee report provides a breakdown of case types by service, while the last section documents overall case trends in the OR since the beginning of the year, by month.

\section{Surgeon/Resident/Department Reports}

Two types of reports are produced on a monthly basis for surgeons, surgical residents, and surgical departments. The first is a "log" that provides a chronological record of the operative experience of either the individual or the group of individuals being reported. The second type of report provides a tabulation of the individual surgical procedures listed in the $\log$, with the procedures ordered in decreasing frequency of occurrence. These reports are used by surgeons in applying for board certification, by residents in documenting their operative experiences, by residency program directors in evaluating educational experience, and by department chairmen in evaluating their departments' overall performance.

\section{Undictated/Unsigned Report}

The HFH medical record department (MRD) and the surgical suite provide a combined weekly report regarding the status of completion of medical documentation. This report, which provides data at both the individual physician and department level, includes three major types of documentation: (1) undictated and unsigned operative summaries; (2) undictated and unsigned discharge summaries; and (3) unsigned sections of the medical record. The MRD and the surgical suite use independent inventory procedures to keep track of document status. On a weekly basis, these separate document inventories are entered into the combined reporting system and the reports are produced. Since the data bases contain all reporting data that are required (e.g., patient name; dates of surgery, admission, and discharge; surgical resident; discharging staff physician; discharging resident; the department of physicians and residents), only the OR record numbers (in the case of the OR) and the MRN and admission date (in the case of MRD) need be entered.

\section{Ad Hoc Data Retrieval}

HFH and MISG have jointly developed a sophisticated information storage and retrieval system that provides near-immediate access to multiple data bases, including the ORMIS data base. This retrieval capability, which is used throughout HFH and has hence been dubbed HFHMIS, provides the ability to analyze, stratify, and merge data in multiple ways. HFHMIS facilities include (1) convenient searching, sorting, and tabulation routines; (2) the ability to concatenate records of different types based upon common key field values (e.g., OR and anesthesia records for the same patient); (3) the ability to generate three-color pen-and-ink zip code maps for any patient set that is selected; (4) linkage to an interactive statistical package; and (5) a report generator for formating output that includes English descriptors for ICD-9-CM codes, internal HFH surgical procedure codes, and diagnostic-related groups (DRGs). 
The HFHMIS ad hoc data retrieval capability is used extensively within the surgical suite. Examples of recent applications include (1) generation of day-of-the-week-specific, surgeon-specific utilization data within a particular surgical department to assist in block scheduling; (2) investigation of procedure-specific, day-of-the-week-specific data to justify equipment purchase; (3) evaluation of operation start times on personnel in-service days; (4) analysis of anesthesia base units and time units to help evaluate the impact of proposed changes in anesthesia reimbursement mechanisms; and (5) analysis of emergency surgical patterns to help set night and weekend emergency coverage.

\section{Automated Charge Capture}

The relatively extensive ORMIS data, along with the stringent error detection and control procedures described above, allow automated charging for surgical suite services. At HFH, up to six types of charges are generated for patients (and/or their third-party payors): (1) surgeon professional; (2) anesthesia professional; (3) anesthesia hospital; (4) operating room; (5) recovery room; and (6) supplies.

The first five charge items are generated automatically from the $O R$ and anesthesia record data files while the supplies charges are currently generated manually. Charge generation is performed by a separate software module, which is run on a daily basis after all error correction activities have been completed. Charge module features include control loops to assure that every patient is charged, cross-edits between the OR and anesthesia records to assure that charges are consistent, hard-copy generation of sample bills for manual review by the billers to catch any billing anomalies not detected by the edit routines (a rare occurrence), electronic transfer of charges to the HFH accounts receivable (AR) system, and manual control loops to assure that all OR charges properly clear the AR system.

\section{Reblocking}

One of the key factors impacting on surgical suite utilization is the manner in which cases are "boarded," or advance-scheduled, into the OR. Within teaching hospitals, the scheduling mechanism commonly involves the assignment of blocks of time to particular surgical services and/or individual surgeons. The assigned blocks are periodically evaluated and reassigned, if necessary, to help achieve overall utilization goals.

The existence of comprehensive, valid ORMIS data has greatly increased the ease, both technical and political, with which reblocking is performed within HFH. Correspondingly, overall OR utilization has increased significantly, and the variance in utilization across services has decreased. These improvements are in large part a result of direct surgeon participation in the data capture and reporting processes. Since surgeons are integrally involved in data capture and since they receive monthly feedback on their activity (which they can easily verify), they believe that the data are correct. This belief has resulted in the ability of the OR director to reassign blocks on a quarterly basis in a rational, depoliticized manner. 


\section{DISCUSSION}

In beginning a discussion of the results achieved and lessons learned during development and operation of this system, it is of interest to look at the reasons leading to the initial developmental efforts in 1979. Two forces led to the replacement of an ineffectual batch system with the initial on-line ORMIS system-demands from surgeons to have accurate reports of surgical experience produced annually, and the need to have comprehensive data available to assist in the improvement of basic management systems. Previous attempts to develop improved utilization experience, more effective scheduling algorithms, and improved control of instrument inventories clearly indicated the need for comprehensive, accurate, and timely data on surgical activities.

The version of ORMIS described in this article is considerably different from the initial 1979 version. Many lessons have been learned over the past 4 years, with a number of these leading directly to system enhancements. Perhaps the most important enhancement involved greatly increasing direct surgeon participation in the system. This participation includes both surgeon coding of operative procedures and feedback of complete data to individual surgeons and residents on a frequent (monthly) basis. What is operational here is, of course, a closed feedback loop, which is, as others have rightly noted, the only sure way to guarantee accurate coded data.

An interesting addition to the basic feedback loop experienced with ORMIS is a distinct synergistic effect. Direct surgeon participation has raised medical staff awareness and interest, which has led to further applications, which in turn further raised the need for improved data quality, and so forth. The improvements in data quality were achieved incrementally over time, both through staff participation and through improved data editing, eventually leading to the intrasystem, intersubsystem, and intersystem structure described above. It is believed that edit levels of this type will be increasingly used in conjunction with the development of decentralized systems joined through local area networks.

As expected, many applications have evolved from ORMIS. Included are surgeon and resident reporting, surgical suite utilization and reblocking control, and data to support educational programs, medical quality review, equipment control, and management decision making. The applications just listed are those that could be termed "information-related," in that their value derives from aggregating and reporting data in particular ways. Now that ORMIS has become integrated into ongoing operations and is fully accepted by all surgical suite personnel, we are cycling back to work on the more management-related applications that originally provided a major impetus for system development. Applications currently being pursued include computer-assisted patient scheduling, case cart composition and scheduling, instrument inventory control, and supplies inventory control. While developmental efforts have certainly not been pursued on a full-time basis, it should be noted that 4 years elapsed between the initial decision to proceed with system development and the point of making effective progress on management-related systems. The summary lessons here are that quality data are necessary for effective management systems work, that quality data come from integrated and accepted systems, and that such systems take considerable time to become fully operational. 
The original heavy emphasis on information-related applications led to a keen understanding of the need for an effective ad hoc data retrieval system that can be applied to multiple existing data sets. While fixed format reports are certainly necessary, the ability to further query, analyze, and report data quickly and effectively is an absolute necessity. Writing single-purpose programs to perform this retrieval function is simply too slow and/or too expensive. It has also been learned that personnel who perform the data retrieval must have a sound working knowledge of the data being analyzed and of the question being addressed, and not just a knowledge of the functioning of the data retrieval system itself.

Besides information-related applications, the system performs multiple functions previously handled by clerical personnel. Examples include assuring that all cases are recorded, charging for services rendered and supplies consumed, transferring charges to the accounts receivable system, tracking and reporting on incomplete medical documentation, and periodic reporting of case statistics. Major computer assistance in these activities as well as methods improvements in ongoing procedures have led to an overall reduction of four FTEs within OR clerical personnel, even though these personnel have assumed the data entry and control functions for all OR and anesthesia records. Part of the "integration" of ORMIS has thus involved insertion of the system into the day-today functioning of the surgical suite.

Another area of considerable knowledge growth involves the electronic transfer of data across what could be considered traditional system boundaries. ORMIS data are transferred to the medical records systems, replacing data previously obtained redundantly through the medical records abstracting process. In addition, the surgical suite and the medical records departments cooperate in the weekly reporting of incomplete medical documentation, charge data generated in an automatic fashion by ORMIS are transferred to the accounts receivable system, and surgical suite data are transferred electronically to the concurrent utilization review system for calculation of a working DRG. 'The combined personnel savings from these cross-system transfers total a minimum of three FTEs, and overall data quality has increased significantly.

While cross-system linkages produce benefits, it must be noted that such linkages produce cross-system dependencies as well. When systems are run independently, a backlog in one area usually affects only that area. With linked systems, however, problems in one area can directly affect other areas. Slowness in surgical suite data entry, for example, means that surgical data are unavailable for concurrent utilization review, medical records applications, and billing purposes. This problem is particularly acute with the advent of DRG-based reimbursement, where surgical data significantly impact on the group determination, and hence on review processes and overall reimbursement received. While the integrated nature of ORMIS quickly provides quality surgical data when the system functions as designed, it also places considerable pressure both on the system and on surgical personnel to perform functions in a timely and accurate manner, and penalizes multiple other areas of the institution when either fails to perform to specifications.

HFH has adopted a decentralized philosophy for information systems development. This philosophy calls for the installation of a number of application-specific computers linked through a local area network, and a Sytek Model 20 broad-band cable network is currently being installed. Following this general philosophy, ORMIS will migrate from 
its current main-frame location to an independent, networked machine in the relatively near future. The knowledge gained to date regarding edit levels, cross-system data linkages, cross-system dependencies, and the need to have working knowledge of data for their proper application is of direct use in the development of decentralized, networked systems such as those evolving at $\mathrm{HFH}$.

\section{REFERENCES}

1. Williams, W. L., Improved utilization of the surgical suite, Hospitals 45(5):93-96, 1971.

2. Cresto, J., and Devor, D., Computerize the log. Hospitals 47(13):58-60, 1973.

3. Moll, D., Lande, M., and Buckley, J., O.R. information system implemented. Hospitals 49(1):55-60, 1975.

4. Speller, C. E., and Cliff, K. S., An information system for a surgical unit. Health Soc. Serv. J. 85:21022103, 1975.

5. Rohe, D. K., Operating room statistical information system-ORIS - What an information system should be. Center for Hospital Management Engineering, American Hospital Association, Chicago, 1979.

6. Priest, S. L., Pelati, B. D., and Marcello, D. E., Computerized O.R. log system has many uses. Hospitals 54(11):79-82, 1980.

7. Wasvick, J., and Brislin, J. M., Surgery information system for managing OR utilization. Productivity Improvements in Operating Rooms, An Examination of Case Studies. Proceedings of a Forum, Center for Hospital Management Engineering, American Hospital Association, Tucson, Ariz., 1980, pp. 23-40.

8. Hayes, C. C., and Smith, K. F., A computer information system for the OR suite. AORN J. 33(4):672$676,1981$.

9. Brown, A. C., Computer management of operating room time information with proposed standard definitions for measurement of utilization. Proceedings of the Sixth Annual Symposium on Computer Applications in Medical Care, IEEE, Washington, D.C., 1982, pp. 246-251.

10. Budkin, A., Larsen, P. B., Hunt, D., and Ally, J., On-line management of cardiac surgical data. J. Med. Syst. 3:133-142, 1979.

11. Napoli, J. G., and McGuire, H. P., Operating room computer subsystem for increasing profitability of the surgical suite. Proceedings of the 10th Annual Conference of the Hospital Management Systems Society, Center for Hospital Management Engineering, American Hospital Association, San Diego, 1982, pp. 377-402.

12. Miller, S. P., Automated OR records facilitate university hospital management. Hospitals 56(20):49, 1982. 